\title{
Influence de la fertilisation sur la végétation spontanée et la croissance radiale de l'épicéa commun (Picea abies (L) Karst) dans une plantation adulte des Ardennes françaises
}

\author{
N Fehlen, JF Picard \\ INRA-Nancy, unité d'écophysiologie forestière, 54280 Champenoux, France
}

(Reçu le 6 octobre 1993; accepté le 12 décembre 1993)

\begin{abstract}
Résumé - L'étude dendroécologique d'un dispositif de fertilisation (N, $\mathrm{P}, \mathrm{Ca}, \mathrm{PCa}$ ) installé dans une plantation d'épicéa commun adulte des Ardennes primaires a été entreprise en 1992. Cette plantation, qui reçoit des quantités importantes d'azote d'origine atmosphérique, a présenté à partir de 1986 des symptômes de dépérissement très accentués dans les placettes Témoin et $N$. Cette étude a permis de montrer le rôle essentiel (direct et indirect) joué par l'ion Ca sur l'amélioration de la fertilité stationnelle. Dans les placettes ayant reçu du $\mathrm{Ca}$, on observe : i) une réponse rapide et positive du peuplement à l'amélioration des conditions stationnelles ainsi qu'une meilleure résistance des arbres à la sécheresse ; ii) une augmentation de la diversité floristique (développement du cortège des neutroclines); iii) la transformation de l'humus, qui passe du moder au mull ; iv) le maintien des arbres dans un état sanitaire satisfaisant. Ces résultats ont par ailleurs montré un effet dépressif de l'azote en période sèche sur la vigueur des arbres et ont confirmé sa responsabilité dans l'apparition des symptômes de dépérissement.
\end{abstract}

dendroécologie / fertilisation / dépérissement / épicéa commun / Ardennes

Summary - The effect of fertilization on the natural vegetation and radial growth of Norway spruce (Picea abies (L) Karst) in an adult plantation in the French Ardennes. In 1992, a dendroecological study was carried out in a fertilization experiment (additions of $N, P, C a, P C a$ ) set up in an adult Norway spruce plantation in the French Ardennes. This plantation receives high inputs of atmospheric nitrogen and has exhibited severe decline symptoms in the control and $N$ plots since 1982. This study demonstrates the essential role (direct and indirect) played by the Ca ion in the amelioration of site conditions. In the plots receiving $\mathrm{Ca}$, we observed: $i$ ) a rapid and positive response by the stand to improved site conditions and increased resistance of the trees to drought; ii) an increase in floristic diversity (the development of a neutrophile suite); iii) a transformation of the humus, which changes from a moder to a mull; and iv) a satisfactory state of tree health. These results also show that $N$ has a depressive effect on tree vitality during dry periods and confirm that this element is responsible for the appearance of decline symptoms. 


\section{INTRODUCTION}

En 1980, Nys (1989) a installé un essai de fertilisation dans une pessière adulte des Ardennes afin de déterminer quel(s) élément(s) permettrai(en)t d'augmenter la fertilité de sols intrinsèquement pauvres et donc la production de bois. Les premiers résultats, basés sur des mesures de circonférences, ont montré un effet positif du calcium et négatif de l'azote sur la croissance. En 1986 apparaissent des symptômes de dépérissement (jaunissement et perte d'aiguilles) dont l'intensité est liée à la nature du traitement : les arbres chaulés restent sains tandis que les arbres témoins et ceux ayant reçu de l'azote dépérissent. Ces différences de croissance et d'état sanitaire font appel aux mêmes explications (Nys, 1989). L'apport d'azote ne fait qu'exacerber un phénomène naturel : cet écosystème reçoit de grandes quantités d'azote atmosphérique $(50 \mathrm{~kg} / \mathrm{ha} / \mathrm{an})$ provenant d'élevages (Hollande et Bretagne) et de la pollution urbaine (Paris et Londres) qui engendre de graves déséquilibres nutritionnels (Andersson et Persson, 1988 ; Weissen et al, 1993). L'apport de calcium restaure la fertilité stationnelle par son action directe (levée de la carence calcique) et indirecte (amélioration des propriétés physico-chimiques du sol).

Le chaulage a déjà montré une double vocation : accroître la production et préserver la santé de peuplements susceptibles de développer des déséquilibres nutritifs.

Après ce premier constat, il était intéressant d'analyser plus en détail les réponses du peuplement aux divers apports. Une approche dendroécologique fut adoptée, associant une étude dendrochronologique (basée sur la mesure des cernes d'accroissement annuel) et une étude phytoécologique (basée sur l'analyse de relevés floristiques et la prise en compte de paramètres écologiques).

\section{MATÉRIEL ET MÉTHODES}

\section{Site expérimental}

Le dispositif expérimental a été installé dans un peuplement subadulte d'épicéa commun (Picea abies (L) Karsten) situé en forêt domaniale de Château-Regnault (massif des Ardennes) et issu d'une plantation de 1937 (3250 plants/ha). Au printemps 1992, la densité du peuplement était de 400 tiges par hectare et la hauteur dominante dans le dispositif avoisinait les $25 \mathrm{~m}$

Le site, d'une altitude moyenne de $480 \mathrm{~m}$, passe d'une situation de replat en son tiers supérieur (plateau de la Croix-Scaille) à une légère pente d'exposition sud/sud-est.

Le climat de la haute Ardenne est de type montagnard, froid et humide. La moyenne annuelle des températures s'élève à $7^{\circ} 3$ (station météorologique des Vieux-Moulins de Thillay, située à environ $3 \mathrm{~km}$ ). Les précipitations annuelles sont de l'ordre de $1100 \mathrm{~mm}$, bien réparties dans l'année.

Le sol est de type brun ocreux développé à partir d'un mélange de limons très homogènes d'origine probablement locale et des produits d'altérations des phyllades du Révinien supérieur sous-jacentes (schistes gris-bleu, pauvres en minéraux altérables). L'humus est un moder bien caractérisé.

La végétation climacique se rattache à l'association du Luzulo-Fagetum-vaccinietosum (Drapier, 1989). La plantation de résineux a remplacé un peuplement feuillu composé d'une futaie de chênes sessiles (Quercus petraea (Matt) Liebl) et hêtres (Fagus sylvatica L) dominant un sous-étage de sorbiers des oiseleurs (Sorbus aucuparia L) et bouleaux pubescents (Betula pubescens Ehrh).

\section{Protocole expérimental}

Outre les témoins, 4 traitements ont été testés à la Croix-Scaille :

- N : apport de $200 \mathrm{~kg} / \mathrm{ha}$ d'azote sous forme d'ammonitrate ;

- P : apport de $1250 \mathrm{~kg} / \mathrm{ha}$ de scories Thomas, soit $150 \mathrm{~kg} / \mathrm{ha}$ de $\mathrm{P}_{2} \mathrm{O}_{5}, 500 \mathrm{~kg}$ de $\mathrm{CaO}$, et $75 \mathrm{~kg}$ de $\mathrm{MgO}$;

- Ca : apport de $2500 \mathrm{~kg} / \mathrm{ha}$ de calcaire broyé à plus de $99 \%$ de $\mathrm{CaCO}_{3}$; 
- PCa : apport de $1250 \mathrm{~kg} / \mathrm{ha}$ de scories et 1600 $\mathrm{kg} / \mathrm{ha}$ de calcaire broyé, soit $150 \mathrm{~kg} / \mathrm{ha}$ de $\mathrm{P}_{2} \mathrm{O}_{5}$ et l'équivalent de $2500 \mathrm{~kg} / \mathrm{ha}$ de $\mathrm{CaCO}_{3}$.

L'ammonitrate a été épandu en plein au printemps 1981 et renouvelé en 1983 (100 kg de N/épandage). Les scories et le carbonate de calcium ont été épandus en plein à l'automne 1980 . Les 5 traitements ont été répétés 4 fois, au sein de 4 blocs répartis selon la topographie.

Vingt placettes ont donc été installées. Chaque placette est un carré de $50 \mathrm{~m}$ de côté, traité sur toute sa surface. Seuls les arbres compris dans le carré central de $30 \mathrm{~m}$ de côté sont numérotés et font l'objet de mesures.

\section{Collecte du matériel}

\section{Observations phytosociologiques}

Un relevé classique, suivant la méthode de BraunBlanquet (Braun-Blanquet, 1932), a été effectué sur chaque placette en juillet 1988 . Un relevé a également été fait au sein d'un peuplement feuillu voisin, certainement très proche du peuplement d'origine (placette F).

Ces données floristiques ont été traitées au moyen d'un analyse factorielle des correspondances complétée par une classification ascendante hiérarchique : pour les espèces présentes dans les strates herbacées et arbustives, seule la strate la plus représentée a été prise en compte.

\section{Observations et mesures sur les arbres}

L'état sanitaire de ce peuplement est suivi annuellement depuis le début de l'expérience : en particulier, on a noté l'intensité du jaunissement des houppiers de 10 arbres par placette. Pour la présente étude, nous avons retenus les chiffres de 1992.

Les 702 arbres (35/placette en moyenne) ont été sondés à l'aide d'une tarière de Pressler de $5 \mathrm{~mm}$ de diamètre intérieur à $1,30 \mathrm{~m}$ du sol, parallèlement aux courbes de niveau, à l'est pour les arbres pairs, à l'ouest pour les arbres impairs. Les carottes de sondage ont été prélevées suffisamment tard pour que le cerne de 1991 soit complet.

Après planage (Becker, 1987), les largeurs de cerne d'accroissement annuel ont été mesurées au $1 / 100^{e}$ de millimètre de l'écorce vers le cœur grâce à une chaîne informatisée (Bert et Becker, 1990) et converties en surface terrière (surface de l'anneau correspondant à chaque cerne) afin de mieux tenir compte de la production annuelle de l'arbre. Dans le cas de carottes à cœur (moelle visible), le cerne le plus ancien n'a pas été mesuré (sa largeur varie en fonction de la hauteur de carottage). Dans le cas contraire, on détermine le nombre de cernes manquant à cou en divisant la distance séparant le dernier cerne mesuré du cœur d'une coupe transversale théorique d'arbre par la largeur moyenne des 5 derniers cernes mesurés.

Afin de vérifier la datation parfaite de chaque cerne, chaque dendrochronogramme est interdaté par rapport à une courbe de référence (Bert et Becker, 1990). Dans notre cas, aucune courbe de référence pour l'épicéa dans cette région n'étant disponible, la courbe de référence a été établie à partir de la majorité des arbres Témoins du dispositif ( $n=126$ )

Chaque valeur d'accroissement annuel est ensuite standardisée, c'est-à-dire convertie en indice de croissance ( $\mathrm{l} \%$ ) afin d'éliminer des surfaces de cernes la part de variabilité due à l'effet de l'âge et pouvoir ainsi comparer des cernes élaborés à la même date mais d'âges courants différents (Becker et Lévy, 1988). La présente étude portant sur un peuplement équienne, cette opération n'était pas indispensable ; elle permet cependant de mieux comparer quantitativement des cernes élaborés à des dates différentes.

L'ensemble des profils individuels est stratifié en sous-échantillons qui correspondent à autant de classes d'un paramètre stationnel ou dendrométrique donné. Le dendrochronogramme moyen de chaque sous-échantillon est calculé. Dans les programmes informatiques de stratification existe une procédure permettant, dans les cas où interviennent des traitements, de réajuster les dendrochronogrammes correspondant aux différents traitements par rapport à la croissance de la moyenne des témoins et sur une période de 10 ans précédant immédiatement la date de fertilisation (19711980), ceci afin de rendre plus évident l'influence des traitements sur la croissance radiale.

\section{RÉSULTATS}

\section{Étude phytoécologique}

\section{Tableau floristique}

Cette plantation d'épicéas présente une diversité floristique importante puisque 
50 espèces y ont été répertoriées (tableau I). Onze d'entre elles (espèces "de fond») sont réparties uniformément dans tout le dispositif, quel que soit le traitement. Ces espèces sont en majorité acidiphiles, caractéristiques des sols pauvres en éléments nutritifs et en bases échangeables et de $\mathrm{pH}$ acide ; la plupart sont propres aux humus de type moder (Pteridium aquilinum, Deschampsia flexuosa, Galium saxatile, Carex pilulifera, Luzula luzuloides). Parmi ces espèces, on trouve celles qui sont les plus fréquentes et/ou les plus abondantes dans le dispositif (Deschampsia flexuosa, Galium saxatile, Picea abies G [G signifiant germination (plantules et jeunes plants de moins de $25 \mathrm{~cm}$ environ)], Dryopteris dilatata, Carex pilulifera, Polytrichum formosum). La myrtille est bien représentée dans l'ensemble, mais son importance décroît de la gauche vers la droite du tableau (eutrophisation croissante).

L'apport d'azote entraîne peu de modifications dans le cortège floristique. Les rares espèces qui apparaissent restent très discrètes (coefficient + ). La myrtille se maintient à un fort coefficient, identique aux témoins, et la callune (acidiphile stricte) renforce sa présence.

L'apport de scories et/ou de carbonate de calcium (traitements $\mathrm{P}, \mathrm{Ca}$ et $\mathrm{PCa}$ ) entraîner une forte augmentation de la diversité floristique, plus soutenue pour les traitements $\mathrm{Ca}$ et Pca. De nombreuses espèces apparaissent : acidiclines (Potentilla erecta, Epilobium montanum, Galeopsis tetrahit), neutroclines à large amplitude (Dryopteris filix-mas, Senecio fuchsii, Salix caprea G), neutronitroclines (Taraxacum officinale, Mycelis muralis) et neutronitrophiles (Urtica dioica). Les espèces de mull se renforcent (Rubus idæus, Athyrium filixfemina, Epilobium angustifolium) tandis que celles de moder (Betula pubescens, Vaccinium myrtillus) se raréfient, davantage d'ailleurs dans les placettes $\mathrm{Ca}$ et PCa.

La plantation d'épicéas (placettes témoins) et le peuplement feuillu présentent une végé- tation spontanée globalement identique (cortège d'acidiphiles de moder composé notamment des 11 espèces «de fond»). Toutefois, dans les placettes $T$, on observe une augmentation des espèces acidiphiles de moder (Galium saxatile et surtout Vaccinium myrtillus) ainsi que d'une espèce de mull mésotrophe sur sol plus ou moins désaturé (Dryopteris dilatata) et de semis d'épicéas. À l'inverse, d'autres espèces se raréfient (Deschampsia flexuosa, Carex pilulifera, Luzula luzuloides) tandis que la callune, acidiphile de mor-moder, fait une discrète apparition.

\section{Analyse multivariable}

Les résultats présentés proviennent de calculs basés sur l'abondance-dominance des espèces. L'analyse définitive ne tient pas compte des espèces rares (rencontrées une seule fois sur l'ensemble des relevés).

La connaissance préalable de l'écologie des espèces rencontrées (Rameau et al, 1989) permet de mettre en évidence la responsabilité de facteurs écologiques dans la répartition des espèces (fig 1 ) et/ou des relevés (fig 2 ) selon un gradient le long des axes de projection. Seuls les 2 premiers axes ont pu être interprétés. Les coordonnées des placettes le long de ces axes servent d'indices floristiques (If) lors des stratifications.

\section{Axe 1}

Les espèces se répartissent entre 2 pôles. À une extrémité de l'axe se situent les espèces acidiphiles de moder (Deschampsia flexuosa, Pteridium aquilinum, Vaccinium myrtillus...). À l'autre extrémité se situent les espèces acidiclines (Moehringia trinervia, Galeopsis tetrahit, Luzula campestris...), neutroclines (Senecio fuchsii, Salix caprea...), neutronitroclines (Dryopteris filixmas, Mycelis muralis, Taraxacum officinale...), et neutronitrophiles (Urtica dioica).

Le long de ce premier axe, les relevés se répartissent en 3 groupes. Les placettes 
Tableau I. Composition floristique du dispositif expérimental.

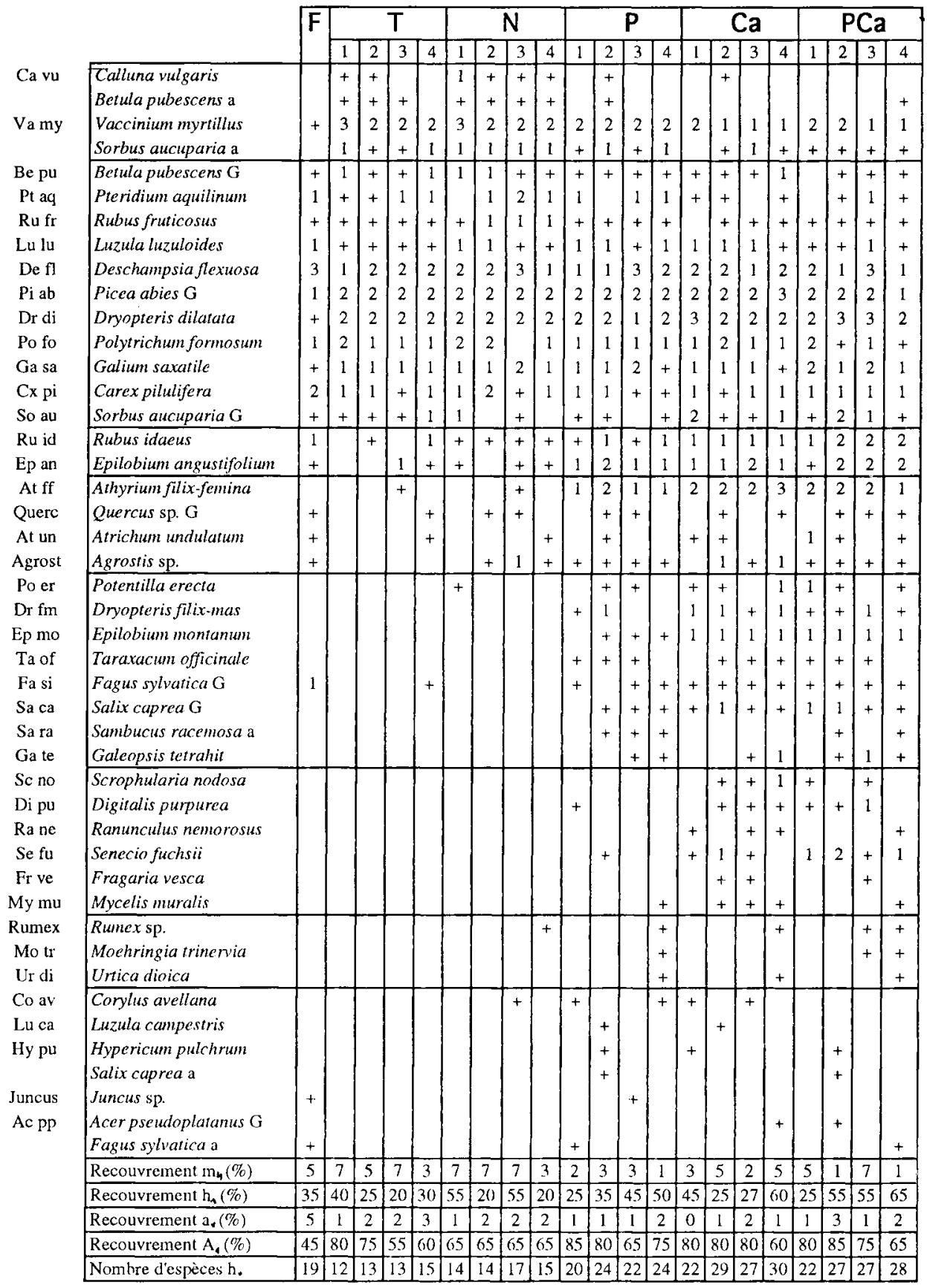

F est la placette "feuillus"; $T, N, P, C a$ et $\mathrm{PCa}$, les différentes modalités de fertilisation. $m$ : mousse, $h$ : herbe a : arbuste, $A$ : arbre, $G$ : semis. Seules figurent dans ce tableau les espèces présentes dans 2 relevés at: moins. 


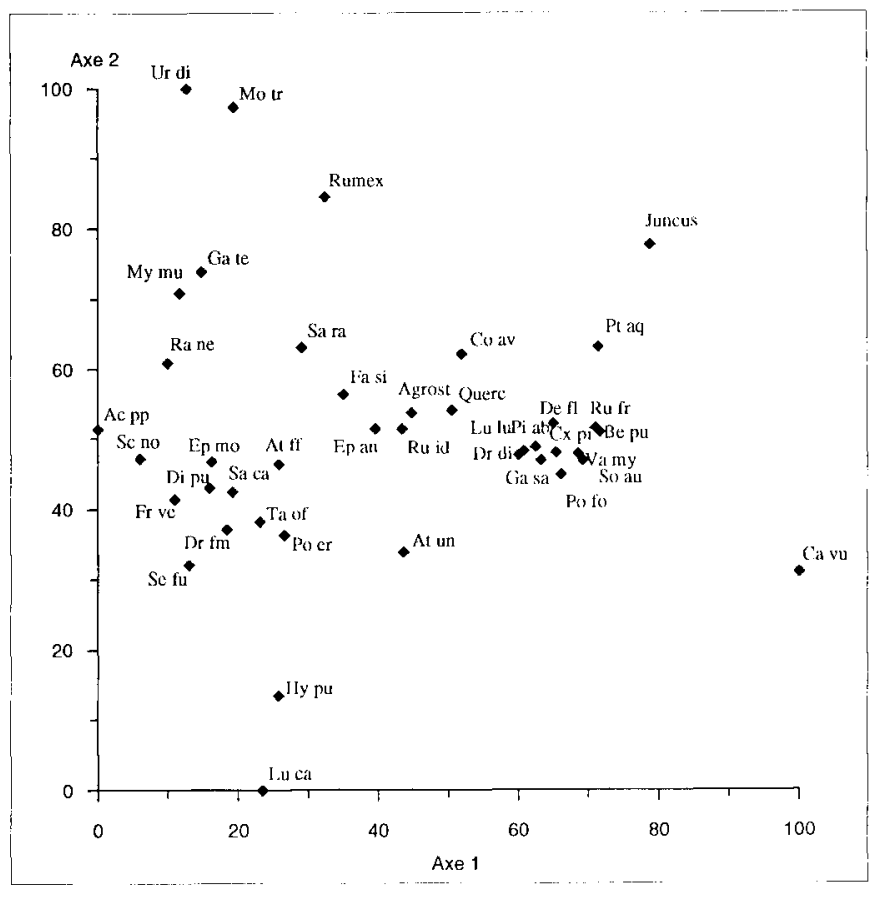

Fig 1. Projection des espèces dans le plan de I'AFC formé par les axes 1 et 2 . La signification des noms d'espèces est donnée dans le tableau I.

T et $N$ correspondent au pôle acide de l'axe ; les placettes $\mathrm{Ca}$ et $\mathrm{PCa}$, au pôle neutrocline. Les placettes $P$ se situent en position intermédiaire. La placette $F$ prend place dans le nuage des placettes $T$ et $N$, mais sur la gauche, en position excentrée (légèrement moins acide).

D'après ces observations, l'axe 1 peut être interprété comme reflétant un gradient trophique au sein du dispositif.

\section{Axe 2}

À l'intérieur des 3 groupes mis en évidence le long de l'axe 1, les placettes se répartissent selon leur appartenance aux différents blocs du dispositif. Cet axe peut être interprété comme reflétant un gradient d'alimentation en eau (profondeur du sol croissante du bloc 1 au bloc 4, liée à leur position topographique) et de richesse (augmentation des réserves échangeables).

\section{Étude dendrochronologique}

\section{Tendance générale}

La dépression observée entre 1971 et 1980 (fig 3), décrite par de nombreux auteurs sur l'épicéa commun (Eichkorn, 1985 ; Perrier, 1989 ; Bouchon, 1989) et le sapin pectiné (Becker, 1987 ; Bert et Becker, 1990), est liée à la période sèche concomitante dont le paroxysme se situe en 1976 . Toutefois, pour $54 \%$ des arbres, 1977 correspond au paroxysme de la crise, preuve manifeste de l'existence d'arrière-effets climatiques sur la croissance, phénomème déjà mis en évidence pour le sapin (Becker, 1987). La reprise intervient dès l'année suivante et, en 1980 , la croissance a retrouvé son niveau initial. Sur la période 1980-1991, le niveau de croissance, bien que supérieur à la moyenne, enregistre une seconde dépression centrée sur 1984-1987. Cette période 
Fig 2. Projection des relevés dans le plan de I'AFC formé par les axes 1 et 2.

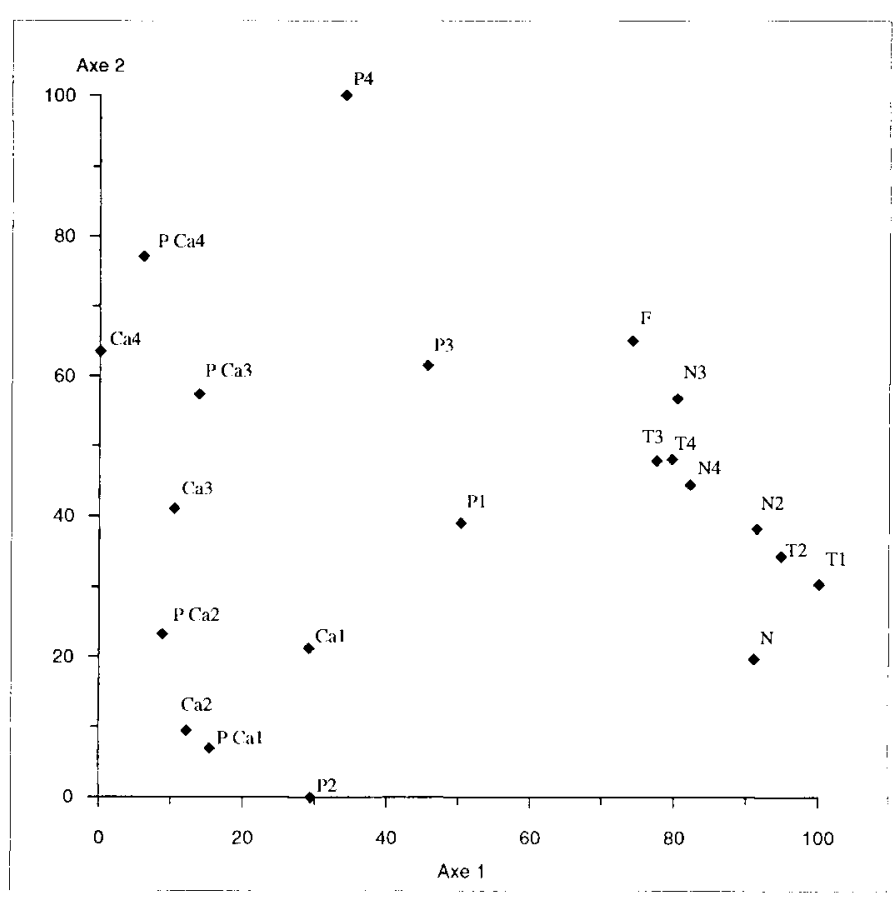

En tenant compte du phénomène d'arrièreeffet des précipitations sur la croissance radiale (déjà mis en cause dans le cas de la crise des années 1970), il est probable que ces sécheresses n'aient réellement eu un impact sur la croissance qu'un ou 2 ans plus tard, de 1984 à 1986-1987.

\section{Effets moyens des traitements}

Suite à l'application de la fertilisation (en 1981), les traitements se divisent en 2 groupes selon la réaction des arbres (fig 4) : d'une part, l'ensemble T-N ; d'autre part, l'ensemble P-Ca-PCa. L'apport d'ammonitrate a un effet dépressif peu marqué sur la croissance radiale par rapport à $T(-10 \%)$ et significatif uniquement pour les années 1987 et 1988 (au seuil de 5\%). L'application des traitements $\mathrm{P}, \mathrm{Ca}$ ou $\mathrm{PCa}$ entraîne une augmentation de la vigueur des arbres par rapport aux témoins dès 1983 (au seuil de 5\%). remarque que 1983,1984 et 1985 ont été
relativement sèches, particulièrement en été. gine dans les facteurs climatiques. En effet, si l'on observe les variations de précipitations au cours de ces dernières années, on 
Toutefois, si l'on affine l'analyse statistique, au seuil de $1 \%$ apparaissent des différences de comportement entre les arbres appartenant à ces différents traitements : $\mathrm{Ca}, \mathrm{P}$ et $\mathrm{PCa}$ divergent par rapport à $\mathrm{T}$ respectivement à partir de 1983, 1984 et 1985.

Le traitement Ca suivi du traitement $\mathrm{PCa}$ et du traitement $P$ (différences non significatives) présente l'augmentation de vigueur la plus forte et la plus précoce, ce qui se traduit par une augmentation de croissance par rapport à la période de préfertilisation de 34,31 et $23 \%$ respectivement. En 1990 et 1991 , le niveau de croissance radiale des arbres $(P)$ chute davantage que celui des arbres (Ca) et (PCa), de telle sorte qu'il rejoint le niveau des Témoins (en 1990 au seuil de $5 \%$ ) sans toutefois rejoindre le niveau du traitement $N$. En parallèle, $P$ diverge brutalement des traitements $\mathrm{PCa}$ et Ca respectivement à partir de 1991 (à $1 \%$ ) et 1990 (à $5 \%$ ), ce qui confirme la hiérarchie au sein de ces 3 traitements.

Les crises de croissance de 1984 et 1987, mises en évidence au niveau de la stratification globale de l'échantillon, sont peu marquées et conservent un caractère ponctuel dans le cas des traitements $\mathrm{P}, \mathrm{Ca}$ et $\mathrm{PCa}$. En revanche, dans le cas des traitements $T$ et $N$, on observe une période de crise très marquée qui s'étale de 1983 à 1988 et connaît son paroxysme en 1987.

Après cette période de crise, les variations de croissance radiale redeviennent synchrones pour tous les traitements, les arbres ( $\mathrm{T}$ ) et $(\mathrm{N})$ ayant toujours une vigueur inférieure à celle des arbres des autres traitements. Toutefois, alors qu'en 1989 les arbres $(T)$ et $(N)$ retrouvent leur niveau de croissance d'avant la crise, les arbres (P), (Ca) et (PCa) atteignent cette même année un niveau de croissance qu'ils n'avaient jamais atteint auparavant. Enfin, tous les traitements présentent une chute de vigueur au cours des 2 dernières années étudiées (1990 et 1991).

\section{État sanitaire et vigueur des arbres}

La stratification selon l'intensité du jaunissement noté en 1992 (fig 5) montre que seuls les arbres qui présentent un état sanitaire très dégradé voient leur croissance affectée (jaunissement supérieur à $45 \%$, rencontré uniquement dans les traitements T et N). Après la crise de 1976, le niveau de croissance de ces arbres est inférieur à celui des arbres plus sains. II n'en diffère toutefois significativement (seuil de 5\%) qu'à partir de 1982-1983 (donc avant l'apparition des

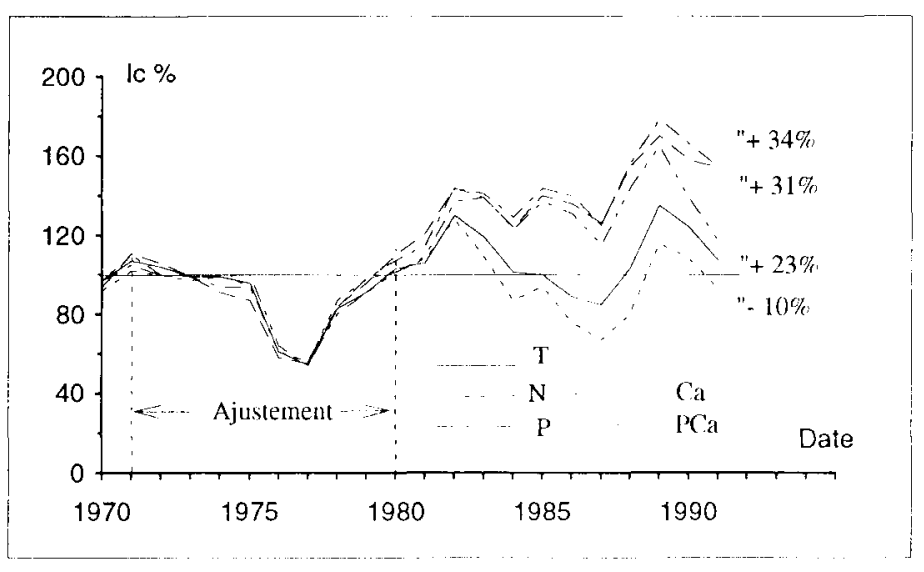

Fig 4. Croissance radiale $(\mathrm{Ic} \%)$ des épicéas de l'ensemble du dispositif de la Croix-Scaille (Ardennes), selon le traitement. 
Fig 5. Croissance radiale $(\mathrm{Ic} \%)$ des épicéas de la CroixScaille (Ardennes), selon le pourcentage d'aiguilles jaunies dans la houppier (notation 1992).

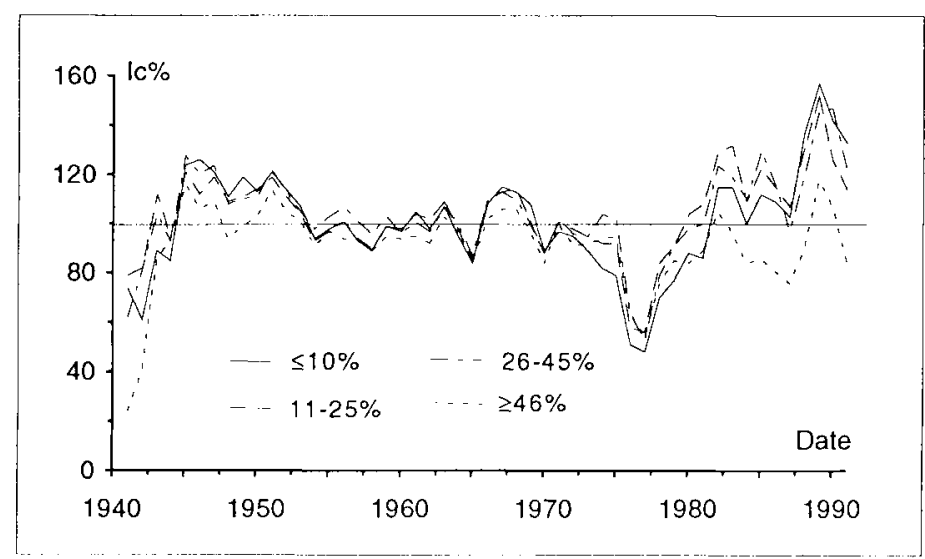

symptômes de "dépérissement"), période pendant laquelle débute la crise mise en évidence au niveau de l'ensemble des arbres.

\section{DISCUSSION ET CONCLUSION}

La position particutière du relevé $F$ le long de l'axe 1 de l'AFC pourrait indiquer un début de dégradation dans les placettes $T$ et $N$, sous épicéa. Elle laisse supposer que l'enrésinement de la station a entraîné une légère détérioration de la fertilité stationnelle, probablement sans conséquence grave à moyen terme. En effet, les analyses physico-chimiques du sol (Nys, 1981) ont montré que, malgré une modification de l'humus (mull acide $\rightarrow$ moder) et du sol (brun acide $\rightarrow$ brun ocreux), 2 à 3 générations d'épicéas ne risquent pas d'altérer la fertilité stationnelle.

L'amélioration de la fertilité dans les placettes P, Ca et PCa, qui se traduit par l'apparition d'un cortège floristique indiquant une certaine eutrophisation du milieu et par l'augmentation de vigueur des arbres de ces mêmes placettes, atteste l'efficacité du calcium apporté. En saturant pour partie le complexe d'échange, ce cation bivalent améliore les propriétés physico-chimiques du sol : le pH est relevé, ce qui entraîne la modi- fication, l'enrichissement et la stimulation de la pédofaune (retour des lombrics) et de la microflore. La minéralisation de la matière organique est accélérée, libérant les éléments nutritifs retenus dans l'humus brut ; ceci, associé à la plus grande disponibilité du calcium et du magnésium, rétablit l'équilibre nutritionnel. L'azote apporté par les dépôts atmosphériques peut être alors utilisé, d'où les symptômes d'eutrophisation. La formation de complexes argilo-humiques est favorisée, ce qui, couplé à la plus grande activité biologique (meilleur brassage des constituants du sol et des différents horizons), améliore la structure du sol et les différents paramètres qui lui sont liés. Le calcium s'était déjà vu attribué ce rôle par Nys (1989) et Belkacem et al (1992) pour ce site, par Becker (1992) et par Lebourgeois (1991) dans différents dispositifs de fertilisation de sapinières vosgiennes.

Le passage de l'humus du type moder au type mull acide dans les placettes $\mathrm{P}, \mathrm{Ca}$ et PCa corrobore le rôle du calcium dans l'amélioration de la fertilité stationnelle, observations faites également par Toutain et al (1988) suite à un amendement dans une hêtraie lorraine.

L'apport de calcium entraîne peu de modifications au niveau du pôle acidiphile, ce qui se traduit par le maintien du cortège 
des espèces de fond qui devront alors être considérées plutôt comme des acidiphiles "tolérantes" (Becker et al 1992).

L'ajout de phosphore au calcium se révèle superflu, le calcium seul conduisant à un meilleur résultat. La position intermédiaire du relevé $P$ le long de l'axe 1 et l'effet à plus court terme du traitement $P$ sur la croissance radiale par rapport à $\mathrm{Ca}$ et $\mathrm{PCa}$ peuvent en effet être expliqués par le calcium apporté par les scories (en quantité moindre que dans le traitement $\mathrm{Ca}$ ).

La grande similitude des placettes $\mathrm{T}$ et $\mathrm{N}$ tant au niveau de la composition floristique que de la vitalité des arbres, alors que dans la majorité des cas l'azote est le facteur limitant de la fertilié stationnelle, peut être expliquée par la pauvreté originelle de la station, en particulier en $\mathrm{Ca}$ et $\mathrm{Mg}$, combinée aux apports massifs d'azote atmosphérique qui peuvent masquer les effets de la fertilisation azotée et rendre celle-ci quasiment insignifiante. Selon Nys (1989), l'azote apporté par les dépôts atmosphériques appauvrit et acidifie encore davantage un sol originellement pauvre en éléments nutritifs. L'ion ammonium $\left(\mathrm{NH}_{4}{ }^{+}\right)$, forme azotée majoritaire dans ces dépôts, est en grande partie transformé en nitrate $\left(\mathrm{NO}_{3}{ }^{-}\right)$qui ne peut être retenu sur le complexe adsorbant : il est lixivié, entraînant avec lui son équivalent cation $\left(\mathrm{Ca}^{2+}, \mathrm{Mg}^{2+} \ldots\right)$. II s'ensuit un appauvrissement du sol, certes, mais aussi des déséquilibres nutritionnels au niveau des végétaux du fait de la trop grande disponibilité de certains éléments par rapport à d'autres. Cette similitude se retrouve au niveau de l'immobilisation des éléments dans les aiguilles et dans le bois qui ne diffèrent pas significativemen entre ces 2 traitements (Belkacem et al, 1992).

La vigueur des épicéas de notre station est peu sensible à la dégradation des houppiers. Selon les régions et les essences, le dépérissement a des origines diverses. Pour Becker (1987), la succession d'années sèches depuis les années 1960 est res- ponsable du dépérissement du sapin pectiné dans les Vosges, hypothèse écartée par Van der Stegen et al (1989) concernant le dépérissement et la perte de vitalité depuis le début des années 1980 d'épicéas situés à la Croix-Scaille (côté belge). Dans le cas de notre site, Nys met en cause les déséquilibres nutritionnels, hypothèse déjà avancée par Bosch et al (1983), Zech et Popp (1983), Zöttl et Mies (1983), Landmann et al (1987) et reprise par Weissen et al (1992) pour d'autres peuplements. En effet, outre l'effet des molécules azotées sur le sol, celles-ci agissent au niveau des aiguilles en provoquant la récrétion des cations basiques (échange entre les molécules azotées et les cations qui sont exsudés). Au niveau des racines, ceux-ci peuvent être réabsorbés par l'arbre mais, pour ce faire, l'arbre excrète des protons, ce qui concentre l'acidité au voisinage des racines. Nos résultats confirment l'hypothèse trophique du dépérissement de notre site : en stratifiant selon l'indice floristique If1 (correspondant à l'abcisse de chaque placette sur l'axe 1 de l'AFC) (fig 6), seuls les arbres (correspondant aux valeurs faibles de l'indice présentent une croissance analogue à celle des arbres très dépérissants; or, ces valeurs correspondent aux placettes $T$ et $\mathrm{N}$; de plus, en tentant de stratifier selon les traitements et l'intensité de dégradation des houppiers, nous nous sommes heurtés à des problèmes d'effectif de classe puisque les traitements $\mathrm{T}$ et $\mathrm{N}$ comprennent principalement des arbres très dépérissants tandis que les arbres $(\mathrm{P})$, (Ca) et (PCa) sont en majorité peu ou pas dépérissants.

Étant donné la différence de comportement entre les arbres du groupe T- $N$ et ceux du groupe $\mathrm{P}-\mathrm{Ca}-\mathrm{PCa}$ durant la période 19841987 , on peut émettre l'hypothèse que l'azote augmente l'effet dépressif des périodes sèches sur la vitalité des arbres tandis que le calcium permet de limiter cet effet. Ces particularités respectives de l'azote et du calcium ont déjà été remarquées par Spiecker (1991) et Becker (1992). II n'est 
Fig 6. Croissance radiale $(\mathrm{Ic} \%)$ des épicéas du dispositif de la Croix-Scaille (Ardennes), selon l'indice floristique If 1 (axe 1 de l'AFC).

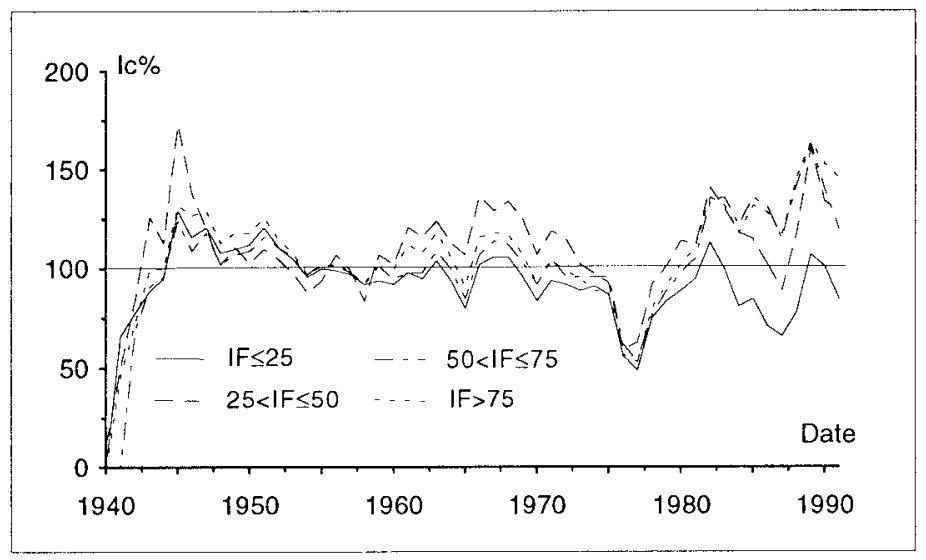

donc pas exclu que les faibles précipitations interviennent comme facteur aggravant de la dégradation de l'état de santé d'arbres dont la nutrition est déséquilibrée.

L'explication de la brutale et importante augmentation de vigueur des arbres $(P)$, (Ca) et (PCa) après la crise de 1987, et de l'exceptionnel niveau de croissance atteint par ceux-ci, reste du domaine de la conjecture. Cette vitalité correspond peut-être à la réponse des arbres vis-à-vis d'une solticitation d'un type nouveau de la part du milieu (augmentation de la concentration en $\mathrm{CO}_{2}$, par exemple), réponse permise par la levée de facteurs limitants (carences nutritionnelles) consécutive à l'application de calcium. Il peut aussi bien correspondre à la réponse directe des arbres suite à l'application de calcium dont les effets auraient été inhibés jusque-là par les années sèches antérieures. II n'est pas impossible que le résultat obtenu provienne de la conjonction de ces 2 phénomènes.

Pour expliquer la chute généralisée de la vigueur des arbres de tous les traitements au cours des 2 dernières années concernées par l'étude, on peut invoquer une nouvelle fois les faibles précipitations de ces années. Mais on peut aussi formuler des hypothèses complémentaires. En effet, en 1992, des observations de l'état sanitaire du peuplement ont montré une augmenta- tion du jaunissement dans tous les traitements, y compris $\mathrm{Ca}$, contrairement à l'intensité des pertes d'aiguilles, qui reste stable. Ce phénomène serait dû à l'apparition d'un nouveau déséquilibre nutritionnel lié cette fois aux faibles quantités de magnésium disponibles [Dambrine et al (1993) ont montré, dans une pessière des Vosges, que la sécheresse tendait à augmenter le jaunissement corrélativement à une réduction du prélèvement de magnésium]. Une expérience complémentaire destinée à verifier cette hypothèse est en cours.

En guise de conclusion, soulignons que l'analyse dendrochronologique a prouvé sa plus grande précision par rapport à d'autres méthodes plus traditionnelles : ce n'est qu'à partir de 1986 (Nys, 1989) que les mesures de circonférence des arbres ont montré un effet significatif de l'apport de calcium.

Par ailleurs, les mesures de circonférence d'une part ont montré un effet très dépressif de l'apport d'ammonitrate sur la croissance et d'autre part attribuent à l'apport de scories seules la meilleure efficacité sur la croissance radiale des arbres. L'étude dendrochronologique a permis de relativiser ces résultats. Un éventuel effet positif du phosphore sur la production d'écorce ou encore le réajustement des courbes sur celle des arbres témoins peuvent être évoqués pour expliquer, au moins 


\section{partiellement, les divergences entre les résultats.}

Enfin, la vitalité des arbres évaluée par les mesures de cernes diverge plusieurs années avant l'apparition des symptômes visuels apparus sur les couronnes à partir de 1986.

\section{RÉFÉRENCES}

Anderson F, Persson T (1988) Liming as measure to improve soil and tree conditions in areas affected by air pollution: results and experiences of ongoing research programme. Report N 3518 , National Swedish Environmental Protection Board, Solna, Sweden, $129 \mathrm{p}$

Becker M (1987) Bilan de santé actuel et rétrospectif du sapin (Abies alba Mill) dans les Vosges. Étude écologique et dendrochronologique. Ann Sci For 44, 379-402

Becker M (1992) Radial growth of mature silver firs (Abies alba Mill) fertilized in 1969. Interaction of climate and competition. Internationai Dendrochronological Symposium "Tree Rings and Environment", Lund, Sweden, 3-9 Sept 1990, Lundqua Report 34, 5 p

Becker M, Lévy G (1988). À propos du dépérissement des forêts : climat, sylviculture et vitalité de la sapinière vosgienne. Rev For Fr 40, 345-358

Becker M, Bonneau M, Le Tacon F (1992) Long-term vegetation changes in an Abies alba forest: natural development compared with response to fertilization. J Veg Sci 3, 467-474

Belkacem S, Nys C, Gelhaye D (1992) Essai d'une fertilisation et d'un amendement sur l'immobilisation d'éléments dans la biomasse d'un peuplement adulte d'épicéa commun (Picea abies L Karst). Ann Sci For 49, 235-252

Bert GD, Becker M (1990) Vitalité actuelle et passée du sapin (Abies alba Mill) dans le Jura. Etude dendroécologique. Ann Sci For 47, 395-412

Bouchon J (1989) Dépérissement et croissance de l'épicéa dans les Vosges. Journées de travail DEFORPA, Nancy-Paris, France, fevrier-mars 1989, vol 1, 3.4.1 $-3.4 .12$

Bosch C, Pfannkuch E, Baum U, Rehfuess KE (1983) Über die Erkrankung der Fichte (Picea abies Karst) in den Hochlagen des Bayerischen Waldes. Forstwiss Centralb/ 102, 167-181

Braun-Blanquet J (1932) Plant sociology (English translation of pflanzensoziologie). Koelz scientific books, reprint $1993,439 \mathrm{p}$

Dambrine E, Carisey N, Pollier B, Granier A (1993) Effects of drought on the yellowingstatus and the dynamics of mineral elements in the xylem sap of declining spruce (Picea abies L). Plant Soil, 150, 303-306
Drapier J (1989) Les stations forestières de l'Ardenne primaire. Écologie, potentialités, catalogue, cartographie. Editions IFN-Nancy, $180 \mathrm{p}+$ annexes

Eichkorn $T$ (1985) Wachstumsanalysen an Fichten in Südwestdeutschland. Allg Forst-Jagdztg 157, 7, 125 138

Landmann G, Bonneau M, Adrian M (1987) Le dépérissement du sapin pectiné et de l'épicéa commun dans le massif vosgien est-il en relation avec l'état nutritionnel des peuplements ? Rev For Fr 39, 1, 5-9

Lebourgeois F (1991) Modifications observées à la suite de la fertilisation dans diverses sapinières dépérissantes des Vosges. Étude phytoécologique et dendrochronologique. Mémoire de DEA en écologie générale et production végétale, université d'Orsay $47 p+$ annexes

Nys $C$ (1981) Modifications des caractéristiques physico-chimiques d'un sol acide des Ardennes primaires par la monoculture d'épicéa commun. Ann Sci For 38, 2, 237-258

Nys C (1989) Fertilisation, dépérissement et production de l'épicéa commun (Picea abies) dans les Ardennes. Rev For Fr 41, 4, 336-347

Perrier P (1989) Étude de la croissance radiale de l'épicéa (Picea abies) sur un échantillon du transect jurassien 2220 : relation avec le phénomène actue du dépérissement forestier. Document Lab Dendrochronologie, Univ Besançon, $25 p$

Rameau JC, Mansion D, Dumé G et al (1989) Flore forestière française. Guide écologique illustré. Tome 1. Plaines et collines. Institut pour le Développement Forestier, Paris, $1785 \mathrm{p}$

Spiecker H (1991) Growth variation and environmental stresses: long-term observations on permanent research plots in southern Germany. Water Air Soil Pollut, 54, 247-256

Toutain F, Diagne A, Le Tacon F (1988) Possibilités de modification du type d'humus et d'amélioration de la fertilité des sols à moyen terme en hêtraie par apport d'éléments minéraux. Rev For Fr 40, 2, 99107

Van der Stegen J, Dutilleul P. Weissen F (1989) Répercussion des symptômes du dépérissement sur la croissance des épicéas. Silva Belg 96, 3, 17-22

Weissen F, Van Praag HJ, Maréchal P, André P (1992) Causes du dépérissement des fôrets en Ardenne: observations et expérimentation. Silva Belg 99, 1 , 9-13

Weissen F, Nys C, Bonneau M (1993) Rỏle des amendements dans la lutte contre le dépérissement des peuplements forestiers. Réunion chaulage, INRA, Nancy, 26 janvier.

Zech $W$, Popp E (1983) Magnesiummangel, einer der Gründe für das Fichten und Tannensterben in NOBayern. Forstwiss Centralbt 102, 50-55

Zöttl HW, Mies (1983) Die Fichtenerkrangung in Hochlagen des Südschwarzwaldes. Allg Forst Zagdztg 154, $6 / 7,110-113$ 